\title{
Who Activates the Nucleophile in Ribozyme Catalysis? An Answer from the Splicing Mechanism of Group II Introns
}

\author{
Lorenzo Casalino, ${ }^{\dagger}$ Giulia Palermo, ${ }^{\dagger}$ Ursula Rothlisberger, ${ }^{\dagger}$ and Alessandra Magistrato*, ${ }^{*}$ \\ ${ }^{\dagger}$ International School for Advanced Studies (SISSA), via Bonomea 265, 34136 Trieste, Italy \\ ${ }^{\ddagger}$ Laboratory of Computational Chemistry and Biochemistry, Institute of Chemical Sciences and Engineering, École Polytechnique \\ Fédérale de Lausanne, CH-1015 Lausanne, Switzerland \\ ${ }^{\S}$ CNR-IOM-Democritos National Simulation Center c/o SISSA, via Bonomea 265, 34136 Trieste, Italy
}

Supporting Information

\begin{abstract}
Group II introns are $\mathrm{Mg}^{2+}$-dependent ribozymes that are considered to be the evolutionary ancestors of the eukaryotic spliceosome, thus representing an ideal model system to understand the mechanism of conversion of premature messenger RNA (mRNA) into mature mRNA. Neither in splicing nor for self-cleaving ribozymes has the role of the two $\mathrm{Mg}^{2+}$ ions been established, and even the way the nucleophile is activated is still controversial. Here we employed hybrid quantumclassical QM(Car-Parrinello)/MM molecular dynamics simulations in combination with thermodynamic integration to characterize the molecular mechanism of the first and rate-determining step of the splicing process (i.e., the cleavage of the 5 -exon) catalyzed by group II intron ribozymes. Remarkably, our results show a new RNAspecific dissociative mechanism in which the bulk water accepts the nucleophile's proton during its attack on the scissile phosphate. The process occurs in a single step with no $\mathrm{Mg}^{2+}$ ion activating the nucleophile, at odds with nucleases enzymes. We suggest that the novel reaction path elucidated here might be an evolutionary ancestor of the more efficient two-metal-ion mechanism found in enzymes.
\end{abstract}

G roup II intron ribozymes (GIIRs) are self-splicing RNAs that are mostly found in bacteria and lower eukaryotes. They play an essential role in gene expression, being in charge of converting premature messenger RNA (mRNA) into mature mRNA. ${ }^{1}$ These RNA macromolecules can autocatalyze their excision from an RNA strand via two distinct transesterification events (i.e., self-splicing; Figure $S 1$ in the Supporting Information (SI)), yielding ligated exons and the intron in a lariat/linear form, or can also undergo a reverse splicing reaction into RNA/DNA filaments, contributing to gene diversification. Remarkably, they are believed to share common evolutionary origins with the eukaryotic spliceosome. ${ }^{2}$ Indeed, it has recently been demonstrated that the RNA moiety of the spliceosome is exclusively in charge of catalysis, with the active site and a two- $\mathrm{Mg}^{2+}$-dependent mechanism similar to those of GIIRs. $^{2 \mathrm{a}, \mathrm{b}}$ Pre-mRNA splicing is a key biological process per se and turns into a critical one considering that its aberrations in humans are responsible for $13 \%$ of genetic diseases and other complex pathologies (cancer and neurodegeneration). ${ }^{2 \mathrm{c}}$ Hence, deciphering the mechanism of splicing at the atomistic level is of the utmost importance, as it may result in revolutionary gene modulation tools and novel therapeutic approaches. ${ }^{2 \mathrm{C}} \mathrm{A}$ breakthrough in the chemistry of GIIRs splicing was provided by a series of crystal structures capturing a group IIC intron at sequential stages of the catalytic process. ${ }^{3}$ These revealed an active site containing a four-metal-ion cluster made of two $\mathrm{Mg}^{2+}$ and two $\mathrm{K}^{+}$ions, the former being catalytically active and the latter likely playing a structural role (Figure 1 and Movie S1).

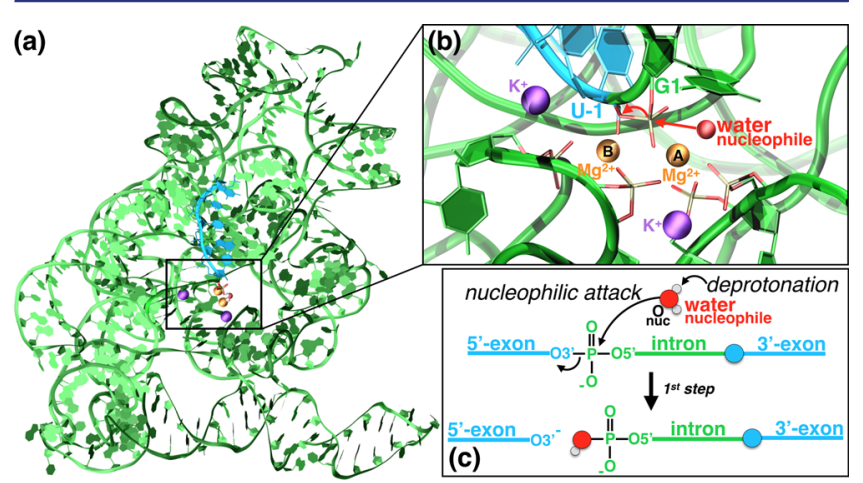

Figure 1. (a) Architecture of the Oceanobacillus iheyensis group IIC intron before splicing ( $\mathrm{PDB}$ entry $4 \mathrm{FAQ}^{3 \mathrm{a}}$ ); the intron and the exon are depicted in green and blue ribbons, respectively. (b) Inset of the catalytic site with $\mathrm{Mg}^{2+}, \mathrm{K}^{+}$, and the nucleophilic water, shown as orange, violet, and red spheres, respectively. The phosphate atoms of the active site are depicted in sticks. (c) Scheme of the first hydrolysis step investigated here.

$\mathrm{Mg}^{2+}$-dependent phosphodiester bond hydrolysis is believed to occur via a two- $\mathrm{Mg}^{2+}$-ion mechanism (Figure S2), as proposed by Steitz and Steitz on the basis of DNA polymerase crystal structures and assumed to be representative also for GIIRs. ${ }^{4}$ In their proposal, the two $\mathrm{Mg}^{2+}$ ions act concertedly as Lewis acids to properly orient and activate the nucleophile and to stabilize the leaving group and the phosphorane transition state/intermediate (Figure S2). This role, which has been confirmed in several computational studies of nucleases, ${ }^{5}$ has not been assessed for two- $\mathrm{Mg}^{2+}$-dependent ribozymes. Moreover, for self-cleaving ribozymes the function and identity of

Received: February 5, 2016

Published: June 16, 2016 
general acid/base contributions are controversial, ${ }^{6}$ mostly because of the lack of RNA residues with unperturbed $\mathrm{p} K_{\mathrm{a}}$ values around $7 .^{7}$ Thus, in computational studies aimed at characterizing their reaction mechanism, a preactivated nucleophile is often used ${ }^{6 a, b}$ or the role of general base is either assigned to hydroxyl ions located in the vicinity of the nucleophile ${ }^{6 c}$ or to nucleobases, sometimes in rare protonation states. $^{6 \mathrm{~d}}$

In this work, we focus on the mechanism of the first and ratedetermining step of the splicing reaction of GIIRs, ${ }^{3}$ in which the 5 '-exon cleavage is mediated by a nucleophilic water molecule (Figures 1 and S1). By using classical and quantumclassical (QM(Car-Parrinello)/MM) molecular dynamics (MD), ${ }^{8}$ with the QM part treated at the DFT-BLYP ${ }^{9}$ level of theory, in combination with thermodynamic integration (see the SI for computational details), we reveal a novel dissociative two- $\mathrm{Mg}^{2+}$-ion mechanism in which the bulk water acts as the general base.

Our simulations are based on the $\mathrm{Ca}^{2+}$-inhibited structure upon replacement of $\mathrm{Ca}^{2+}$ with $\mathrm{Mg}^{2+}$ ions. The reliability of the initial adduct was assessed before the reaction was studied (section 1.4 in the SI and Figures S3-S7). The classical ( 200 $\mathrm{ns})$ and $\mathrm{QM} / \mathrm{MM}(\sim 10 \mathrm{ps}) \mathrm{MD}$ equilibrated reactant shows the putative catalytic water coordinated to $\mathrm{Mg}^{2+}-\mathrm{A}(\mathrm{MgA})^{10}$ and a structural arrangement that is perfectly consistent with catalysis (i.e., the nucleophilic oxygen is in line with the scissile phosphate). However, there is no stable H-bonding network heading from the nucleophile to a putative general base that could activate it, ${ }^{5}$ reconfirming the nontrivial assignment of the general base in ribozyme catalysis. We remark that although the X-ray structure of this GIIR lacks domain VI, which might include a general base, this reduced ribozyme construct is fully active for the hydrolytic pathway investigated here. ${ }^{3}$ To address alternative viable mechanisms, we considered two additional model systems bearing nucleobases in rare protonation states at H-bond distance from the nucleophile: a G1 deprotonated at $\mathrm{N} 1$ and A287 deprotonated at N6. However, 50 ns of classical MD simulation for each model did not lead to a H-bonding network potentially capable of promoting catalysis (Figure S8). Instead, the bases of the D5 bulge (A376-C377) and those for which a $\mathrm{p} K_{\mathrm{a}}$ close to 7 has been reported (here A364-U384) ${ }^{7}$ were not within $\mathrm{H}$-bonding distance from the nucleophile. These may be implicated in the conformational switch occurring after the first step. ${ }^{3 a}$ Finally, replacing the nucleophilic water with a hydroxyl ion resulted in a complete distortion of the catalytic site (Figures S9 and S10), suggesting that a reaction path with a preactivated nucleophile can be ruled out either via canonical or alternative channels. ${ }^{11} \mathrm{We}$ then performed QM/MM blue moon ensemble simulations $(\sim 100$ ps) on the model with the nucleophilic water and nucleobases in standard protonation states, choosing as the reaction coordinate $(\mathrm{RC})$ the difference between the breaking bond distance $\left(\mathrm{O}^{3 \prime} @ \mathrm{U}-1-\mathrm{P} @ \mathrm{G} 1 ; \mathrm{d} 1\right)$ and the forming bond distance $\left(\mathrm{O}_{\text {nuc }}-\mathrm{P} @ \mathrm{G} 1 ; \mathrm{d} 2\right)$, where $\mathrm{O}_{\text {nuc }}$ is the oxygen of the crystallized nucleophilic water (Figure 2a). These simulations unveiled the following mechanism: (i) From $\mathrm{RC}=-0.5$ to $-0.1 \AA$, there is a large increase in the distance between $\mathrm{O}^{\mathrm{Rp}} @$ $\mathrm{G} 1$ and $\mathrm{Mg}^{2+}-\mathrm{B}(\mathrm{MgB})$ and the distance between $\mathrm{O}_{\text {nuc }}$ and $\mathrm{MgA}$ ( $\mathrm{d} 6$ and d4, respectively; Figures $2 \mathrm{~b}$ and 3 and Movie S2). This corresponds to the transfer of the $\mathrm{O}^{\mathrm{Rp}}$ of the scissile phosphate to $\mathrm{MgA}$ and the simultaneous detachment of the nucleophile from $\mathrm{MgA}$, respectively. Thus, a first difference from the protein two- $\mathrm{Mg}^{2+}$-ion mechanism emerges: $\mathrm{MgA}$ does

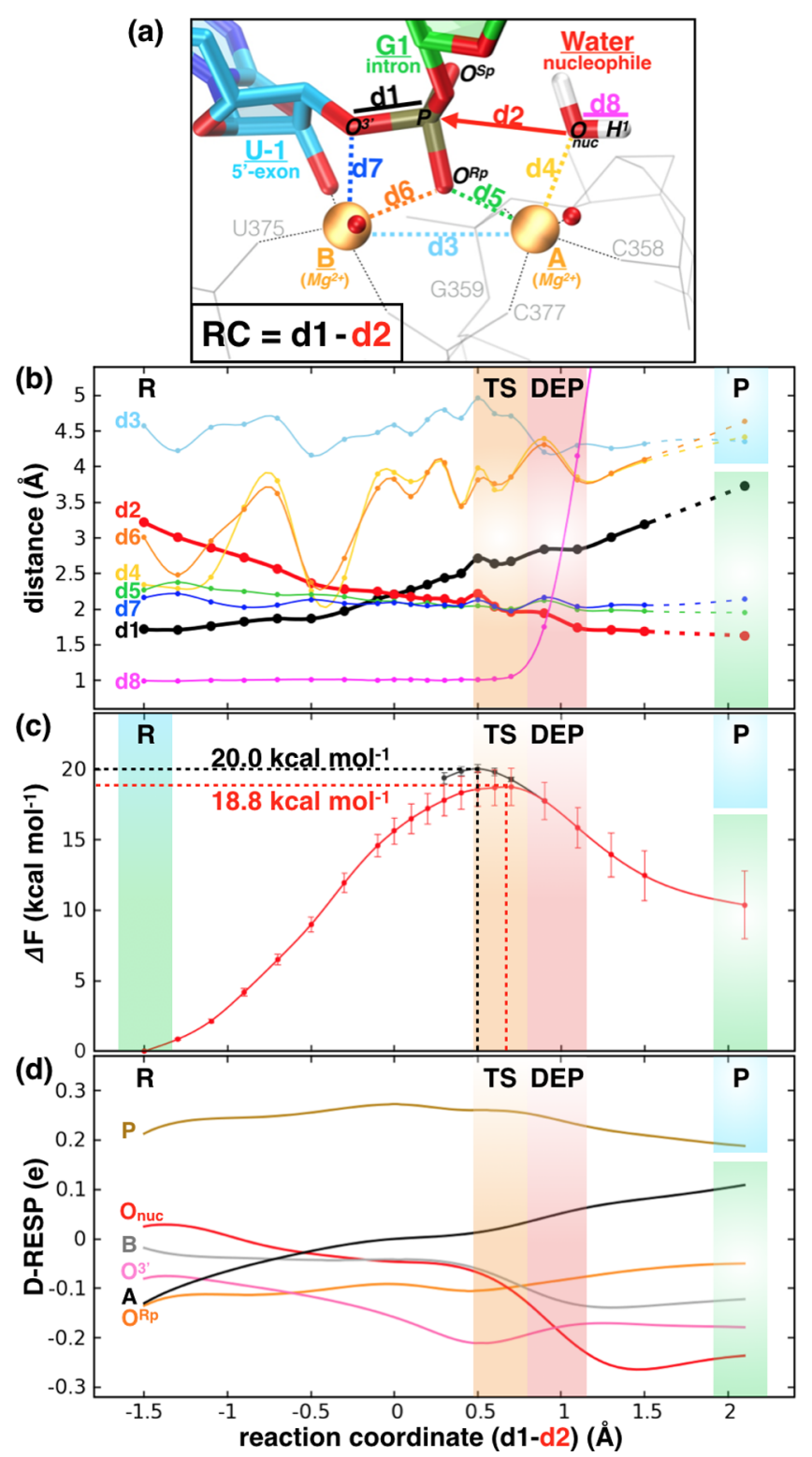

Figure 2. (a) Snapshot of the reactant state showing the reaction coordinate (RC) and the bond distances monitored along the RC in (b). (c) Free energy profiles $(\Delta F$, in $\mathrm{kcal} / \mathrm{mol})$ : red and black lines refer to $\Delta F$ calculated using BLYP $^{9}$ for the direct and reverse processes, respectively. (d) Dynamical RESP (D-RESP) ${ }^{8 \mathrm{~b}}$ charges monitored along the RC. The RC portions corresponding to the reactant state $(\mathrm{R})$, transition state (TS), water deprotonation (DEP), and product $(\mathrm{P})$ formation regions are highlighted with colored areas.

not act as a Lewis acid in activating the nucleophilic water. (ii) At $\mathrm{RC}=0.0 \AA$, before the transition state (TS) is reached, the dissociation of the leaving group $\left(\mathrm{O}^{3 /-} @ \mathrm{U}-1\right)$ from the scissile phosphate (P@G1) takes place (Figure 2b), in line with a dissociative mechanism. Here $\mathrm{MgB}$ facilitates the cleavage by stabilizing the oxyanion leaving group while MgA stabilizes the metaphosphate TS. Although never observed in nucleases, ${ }^{5}$ dissociative cleavage of the phosphodi(mono)ester bond has been reported to occur in other enzymes, such as alkaline phosphatases, ${ }^{12}$ sometimes promoted by a non- $\mathrm{Mg}^{2+}$-coordinated water as in actin or EcoRV. ${ }^{13}$ (iii) At the TS ( $\mathrm{RC}=0.5-$ $0.7 \AA$ ), the bond between $\mathrm{O}_{\text {nuc }}$ and $\mathrm{P} @ \mathrm{G} 1$ starts to form (Figures $2 \mathrm{~b}$ and 3 and Movie S2). In contrast to what is 


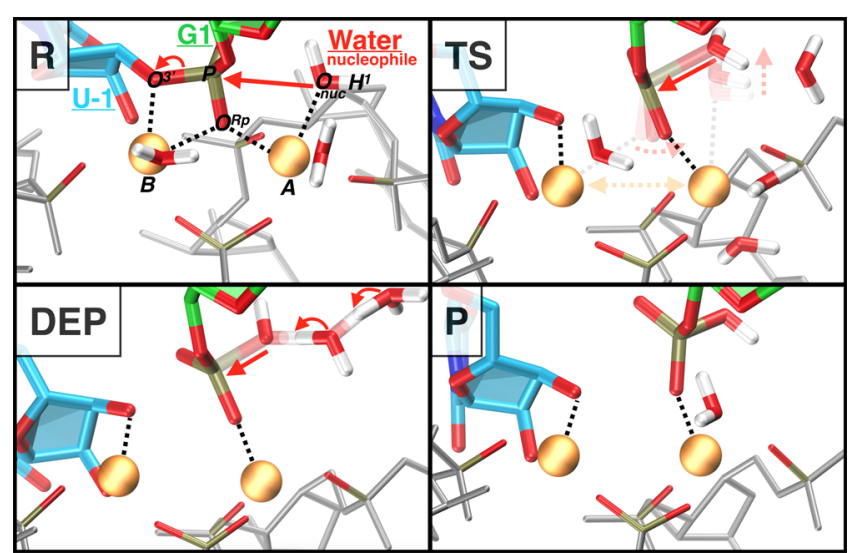

Figure 3. Snapshots of the reactant (R), transition state (TS), deprotonation event (DEP), and product $(\mathrm{P})$. Reactive atoms are depicted in sticks with intron and exon carbon atoms colored in green and blue, respectively; $\mathrm{Mg}^{2+}$ ions are shown in orange, and the rest of the catalytic site is represented in light sticks.

typically found in RNase enzymes, ${ }^{5 a, c}$ at the TS the water is still protonated. (iv) At $\mathrm{RC}=0.9-1.5 \AA$, after $\mathrm{O}^{\mathrm{Rp}} @ \mathrm{G} 1$ moves toward $\mathrm{MgA}$ and the proton of the nucleophile $\left(\mathrm{H}^{1}\right)$ is released to the bulk water (d8; Figure 2b), the $\mathrm{O}_{\text {nuc }}-\mathrm{P} @ \mathrm{G} 1$ bond completely forms. By changing the $\mathrm{QM} / \mathrm{MM}$ partitioning in favor of a more hydrated catalytic site, we observed a proton transfer to the bulk water involving up to five water molecules. (v) At $\mathrm{RC}=2.1 \AA$, formation of the product occurs with structural features resembling those of the corresponding crystal structure (PDB entry 4FAR; ${ }^{3 a}$ Figure S6). It should be noted that the leaving group ( $\mathrm{O}^{3 \prime-} @ \mathrm{U}-1$ of the $5^{\prime}$-exon) retains a negative charge (stabilized by coordination to $\mathrm{MgB}$ ) to act as nucleophile in the second step of splicing (Figure S1).

This process occurs with a Helmholtz free energy barrier $\left(\Delta F^{\ddagger}\right)$ of $18.8 \pm 1.5 \mathrm{kcal} / \mathrm{mol}$ (Figure $2 \mathrm{c}$, section 1.5 in the SI, and Figure S11), in line with the experimental catalytic rate of $0.011 \mathrm{~min}^{-1}$, 3 a corresponding to a $\Delta G^{\ddagger}$ value of $\sim 23 \mathrm{kcal} / \mathrm{mol}$. We remark that the measured rate constant also includes the slow $\mathrm{Mg}^{2+}$-dependent folding of GIIR, thus providing an upper limit to the splicing kinetics. Moreover, the calculated barrier is just slightly higher than the one calculated for RNase $\mathrm{H}$ with the same computational approach. ${ }^{5 \mathrm{a}}$ Additional control calculations performed on small model systems of the catalytic site for the reactant (R) and transition state (TS) using the $\mathrm{B} \mathrm{LYP}^{9 \mathrm{~b}, 14}$ and $\mathrm{BLYP}^{9}$ functionals and a localized basis set (section 1.5 in the SI), led to $\Delta G^{\ddagger}$ values of $\sim 25$ and $\sim 19 \mathrm{kcal} /$ mol, respectively, suggesting that BLYP slightly underestimates the barrier. To further check the trend of the free energy profile within the water deprotonation window (DEP), we also performed constrained QM/MM MD simulations with B3LYP for selected RC points. This confirmed that no additional barrier is associated with the proton transfer event, with the TS lying at the same RC value (section 1.5 in the SI and Figure S11).

To establish the functional role of the observed structural rearrangements, we have plotted the dynamical RESP ${ }^{8 \mathrm{~b}}$ (DRESP) charges along the RC (Figure 2d). These were dynamically calculated during our $\mathrm{QM} / \mathrm{MM} \mathrm{MD}$ simulations, allowing us to monitor the changes in the chemical state of the system along the reaction. This analysis reveals an increase in the P@G1 charge and a decrease in the $\mathrm{O}^{3 \prime} @ \mathrm{U}-1$ charge just before the TS is reached, in line with a dissociative mechanism.
At the TS, the $\mathrm{O}_{\text {nuc }}-\mathrm{P} @ \mathrm{G} 1$ bond (d2) starts to form, and consequently, the charge on $\mathrm{P} @ \mathrm{G} 1$ decreases again, while that on $\mathrm{O}^{\mathrm{Rp}} @ \mathrm{G} 1$ maintains an increasing trend as $\mathrm{O}^{\mathrm{Rp}} @ \mathrm{G} 1$ progressively moves toward $\mathrm{MgA}$ and detaches from $\mathrm{MgB}$. Finally, after the TS, the nucleophilic water becomes a hydroxyl group by releasing its proton $\left(\mathrm{H}^{1}\right)$ to the bulk and completely forming the $\mathrm{O}_{\text {nuc }}-\mathrm{P} @ \mathrm{G} 1$ bond. This dissociative mechanism clearly points to a distinct catalytic process from the canonical two- $\mathrm{Mg}^{2+}$-ion mechanism observed in enzymes. ${ }^{5}$ Here $\mathrm{MgA}$ activates the electrophile rather than the nucleophile, strongly contributing also to the stabilization of the metaphosphate at the TS, while the water is converted into an $\mathrm{OH}^{-}$group only after the formation of the $\mathrm{O}_{\text {nuc }}-\mathrm{P} @ \mathrm{G} 1$ bond has started. In this manner, the proton release to the bulk occurs with no barrier and without the need of a specific base. To the best of our knowledge this is the first mechanistic study suggesting the bulk water as a possible proton acceptor, offering a proposal for the controversial assignment of the general base in ribozymes.

In summary, our study unveils a dissociative mechanism in which the role of $\mathrm{Mg}^{2+}$ ions remarkably differs from that reported for most enzymes. We believe that this novel mechanism is specific for the ribozyme scaffold. In fact, in enzymes the active site is shaped to host a specific substrate and each residue has a functional role to enhance catalysis. Conversely, in ribozymes the catalytic site is formed by the RNA sugar-phosphate backbone, whose specificity for the reaction is lower than that of amino acids. We have shown here that ribozymes, considered the evolutionary ancestors of enzymes, can perform phosphodiester hydrolysis almost as effectively as the latter by adapting the mechanism to their less specific RNA scaffold. This mechanism opens a new scenario concerning the identity of general acid/base in ribozymes, which will stimulate further experimental and computational studies.

\section{ASSOCIATED CONTENT}

\section{Supporting Information}

The Supporting Information is available free of charge on the ACS Publications website at DOI: 10.1021/jacs.6b01363.

Computational details of the calculations and Figures S1-S11 (PDF)

Movie S1 showing the GIIR structure (AVI)

Movie S2 showing the mechanism (AVI)

\section{AUTHOR INFORMATION}

\section{Corresponding Author}

*alessandra.magistrato@sissa.it

\section{Notes}

The authors declare no competing financial interest.

\section{ACKNOWLEDGMENTS}

We thank ISCRA (Grant HP10BPN8BA) for computational resources. U.R. acknowledges the Swiss National Computing Center for computing resources. LC thanks European Social Fund 2007/2013, Project DOCTOR EUROPAEUS. We thank Lorenzo Casalino for the cover art.

\section{REFERENCES}

(1) Lambowitz, A. M.; Zimmerly, S. Cold Spring Harbor Perspect. Biol. 2011, 3, a003616.

(2) (a) Fica, S. M.; Tuttle, N.; Novak, T.; Li, N.-S.; Lu, J.; Koodathingal, P.; Dai, Q.; Staley, J. P.; Piccirilli, J. A. Nature 2013, 503, 
229. (b) Hang, J.; Wan, R.; Yan, C.; Shi, Y. Science 2015, 349, 1191.

(c) Papasaikas, P.; Valcarcel, J. Trends Biochem. Sci. 2016, 41, 33.

(3) (a) Marcia, M.; Pyle, A. M. Cell 2012, 151, 497. (b) Marcia, M.; Somarowthu, S.; Pyle, A. M. Mobile DNA 2013, 4, 14.

(4) (a) Steitz, T. A.; Steitz, J. A. Proc. Natl. Acad. Sci. U. S. A. 1993, 90, 6498. (b) Boero, M.; Tateno, M.; Terakura, K.; Oshiyama, A. J. Chem. Theory Comput. 2005, 1, 925. (c) Sgrignani, J.; Magistrato, A. J. Phys. Chem. B 2012, 116, 2259.

(5) (a) De Vivo, M.; Dal Peraro, M.; Klein, M. L. J. Am. Chem. Soc. 2008, 130, 10955. (b) Palermo, G.; Cavalli, A.; Klein, M. L.; AlfonsoPrieto, M.; Dal Peraro, M.; De Vivo, M. Acc. Chem. Res. 2015, 48, 220. (c) Rosta, E.; Nowotny, M.; Yang, W.; Hummer, G. J. Am. Chem. Soc. 2011, 133, 8934. (d) Sgrignani, J.; Magistrato, A. ACS Catal. 2015, 5, 3864.

(6) (a) Ganguly, A.; Thaplyal, P.; Rosta, E.; Bevilacqua, P. C.; Hammes-Schiffer, S. J. Am. Chem. Soc. 2014, 136, 1483. (b) Wong, K. Y.; Lee, T. S.; York, D. M. J. Chem. Theory Comput. 2011, 7, 1. (c) Mlynsky, V.; Walter, N. G.; Sponer, J.; Otyepka, M.; Banas, P. Phys. Chem. Chem. Phys. 2015, 17, 670. (d) Zhang, S.; Ganguly, A.; Goyal, P.; Bingaman, J. L.; Bevilacqua, P. C.; Hammes-Schiffer, S. J. Am. Chem. Soc. 2015, 137, 784.

(7) Pechlaner, M.; Donghi, D.; Zelenay, V.; Sigel, R. K. Angew. Chem., Int. Ed. 2015, 54, 9687.

(8) (a) Laio, A.; VandeVondele, J.; Rothlisberger, U. J. Chem. Phys. 2002, 116, 6941. (b) Laio, A.; VandeVondele, J.; Rothlisberger, U. J. Phys. Chem. B 2002, 106, 7300.

(9) (a) Becke, A. D. Phys. Rev. A: At., Mol., Opt. Phys. 1988, 38, 3098. (b) Lee, C.; Yang, W.; Parr, R. G. Phys. Rev. B: Condens. Matter Mater. Phys. 1988, 37, 785.

(10) Boero, M.; Ikeda, T.; Ito, E.; Terakura, K. J. Am. Chem. Soc. 2006, 128, 16798.

(11) Boero, M.; Terakura, K.; Tateno, M. J. Am. Chem. Soc. 2002, 124, 8949.

(12) (a) Lopez-Canut, V.; Roca, M.; Bertran, J.; Moliner, V.; Tunon, I. J. Am. Chem. Soc. 2010, 132, 6955. (b) Nishino, T.; Morikawa, K. Oncogene 2002, 21, 9022.

(13) (a) Akola, J.; Jones, R. O. J. Phys. Chem. B 2006, 110, 8121. (b) Imhof, P.; Fischer, S.; Smith, J. C. Biochemistry 2009, 48, 9061.

(14) Becke, A. D. J. Chem. Phys. 1993, 98, 5648. 